\title{
基于加工意图的机加工艺知识重用方法研究
}

\author{
常智勇 ${ }^{1}$ 陶礼尊 ${ }^{1}$ 李佳佳 $^{2}$ 席增飞 1 \\ (1. 西北工业大学现代设计与集成制造技术教育部重点实验室 西安 710072; \\ 2. 西安航天发动机厂 西安 710100)
}

\begin{abstract}
摘要: 为了解决目前工艺知识重用方法存在的问题, 强化工艺知识重用时工艺员的主观能动性, 满足工艺员对工艺知识的个 性化、多元化需求, 以 MBD 工序模型几何演变过程中蕴含的工艺知识为研究对象, 提出基于加工意图的工艺知识重用方法。 给出了加工意图的建模方法, 加工意图的几何演变、工艺语义信息的捕捉方法以及加工意图的降维处理方法。提出多属性融 合的加工意图与 MBD 工艺知识元的相似性加权评价方法, 并进行了实例验证。
\end{abstract}

关键词: 工艺知识; 加工意图; 知识重用; 相似性度量

中图分类号: TG156

\section{The Measure and Search Method of Process Knowledge Element Based on Machining Intent}

\author{
CHANG Zhiyong ${ }^{1} \quad$ TAO Lizun $^{1} \quad$ LI Jiajia $^{2} \quad$ XI Zengfei $^{1}$ \\ (1. Key Laboratory of Contemporary Design and Integrated Manufacturing Technology of \\ Ministry of Education, Northwestern Polytechnical University, Xi'an 710072;
}

2. Xi'an Space Engine Factory, Xi'an 710100)

\begin{abstract}
In order to resolve the problems of current process knowledge reuse methods, strengthen the initiative and meet the personalized, diversified demands of crafts in the research of process knowledge reuse process, process knowledge contained in geometric evolution of MBD process model is as an object, process knowledge reuse method based on machining intent is proposed. Modeling methods, geometric evolution and process semantic information capture methods, dimensionality reduction method of machining intent are given. Finally, a integration of multi-attribute similarity measurement method between machining intent and MBD process knowledge unit is proposed, the example of verification is also given.
\end{abstract}

Key words: process knowledge; machining intent; knowledge reuse; similarity measurement

\section{0 前言}

工艺知识对现代企业快速开发新产品、保证产 品质量、增强创新能力和提高综合竞争力具有重要 的影响, 是企业重要的智力资源 ${ }^{[1]}$ 。而基于 MBD(Model based definition)技术的工艺设计方法 使得 CAPP 三维化成为了必然的趋势。因此, 在 MBD 三维工艺环境下, 开展应用效能更高的工艺知 识重用方法, 就变的迫切且具有重要的现实意义。

目前, 知识重用的方法主要包括基于文本的检 索和基于内容的检索。基于文本的检索是采用文字

* 国家自然科学基金(51375395)、中国博士后科学基金(2014M552484)和陕 西省自然科学基础研究计划(2016JM5040)资助项目。20161225 收到初 稿, 20170826 收到修改稿
或文字内涵的语义作为检索条件, 在工艺设计的知 识重用中有广泛应用。但文本属于低维信息，作为 检索条件不能完整表达高维的知识需求, 因此基于 内容的检索方式逐渐成为研究热点。基于内容的检 索主要包括基于形状检索和基于标注语义的检索。 具体到机加工艺知识的重用领域，基于形状和基于 拓扑结构的检索方式成为了主流。利用频谱分析、 图论 ${ }^{[2-3]}$ 、降维等方法将零件模型或加工特征依据形 状或者拓扑转化为可度量的数学模型, 在 “相似结 构有相似工艺” 的假设下比较零件模型与已有工艺 模型之间的相似程度以检索相似的工艺实例。虽然 几何结构可能蕴含比文本更高维的知识需求, 但其 粒度大、难以反映设计者对局部特征的多元化知识 需求; 基于标注语义的检索方式将几何结构与其上 蕴含的工艺语义标注信息相结合, 形成了更高维的 
检索方式 ${ }^{[4]}$, 但仍未解决知识粒度大的问题。近年 来, 有学者提出了零件工序模型序列的几何演变信 息才是工艺知识的直接载体这一基本观点, 并探讨 了几何演变驱动下的零件级的工艺相似性度量方 法 $^{[5]}$ 和特征级的工艺相似性度量方法 ${ }^{[6]}$ 。这些方法 虽然将工艺知识细化到了零件结构特征形成过程中 的几何演变环节, 解决了传统方法总是将工艺知识 与零件最终结构相固化而带来的知识粒度较大的问 题, 但总的来说还停留在被动的知识重用层面上, 忽视了设计者的主观需求也是影响工艺重用的一个 重要因素, 进而可能导致知识的重用效能降低。

事实上, 机加工艺设计的目标是由毛坏到零件 产品的动态过程, 设计者的隐性加工意图也蕴含在 对加工动态过程的构思中 ${ }^{[7]}$, 这种更高维的加工意 图显然比上述研究都更能反映工艺知识检索的本质 需求。为了解决目前工艺知识重用方法存在的问题, 本文以 MBD 工艺模型几何演变过程中蕴含的工艺 知识为对象, 从加工过程的几何演变及其所附带的 工艺语义这两个角度给出了 MBD 工艺知识元的表 达形式, 并进一步研究以加工意图作为检索条件的 工艺知识重用新模式。

\section{1 基于加工意图的工艺知识重用方法}

\subsection{MBD 工艺知识元}

本文以 MBD 工序模型几何演变过程中蕴含的 工艺知识为研究对象。如图 1 所示, 以具有完备工 艺信息的 MBD 工序模型为基础, 捕捉工序模型动 态几何演变过程中蕴含的高维工艺信息, 具体则包 括工序模型的几何演变信息及其所附带工艺语义信 息。然后再对这些捕捉到的高维工艺信息进行降维 处理，进而得到低维、非线性的工艺知识。

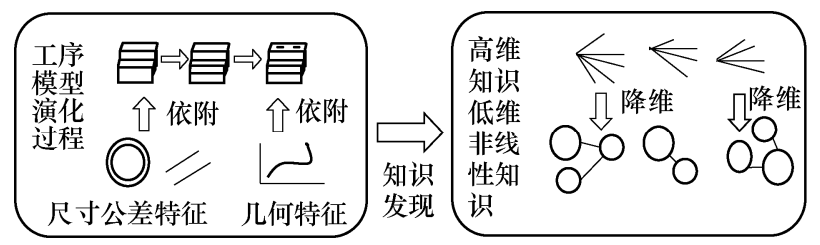

图 $1 \mathrm{MBD}$ 技术的工艺知识发现过程

将工艺知识细化到零件特征结构形成过程中的 几何演变环节, 使知识不再与零件或其上的结构特 征相固结, 知识的粒度明显减小, 知识在重用时也 更加具有灵活性和适用性 ${ }^{[8]}$ 。工艺知识元与零件加工 过程中的一道工序相对应, 表示的是该道工序的基 本工艺内容。零件的机加工艺过程可以看作是由若 干个工序按照一定的顺序排列组成, 即由若干个工 艺知识元按照零件加工的逻辑顺序组织排列得到。
本文从 MBD 工序模型几何演变过程中蕴含的 工艺知识角度出发, 定义工艺知识的基本单元一 MBD 工艺知识元。MBD 知识元解决了知识粒度大 的问题, 是后续工艺知识重用方法研究的基础。

MBD 工艺知识元(Process knowledge unit, PKU) 的矢量表达形式

$$
\mathrm{PKU}=[I D, G, P]
$$

式中, $I D$ 为工艺知识元在工艺知识库中的编号; $G$ 为相邻两道工序模型的几何演变信息; $P$ 为 $\mathrm{MBD}$ 工艺知识元的工艺语义信息。

$$
G=p_{m-i}-p_{m-i+1}
$$

式中, $p_{m-i}$ 和 $p_{m-i+1}$ 分别为第 $i$ 道工序的工序模型与 第 $i+1$ 道工序的工序模型。

$P$ 是从 MBD 工序模型中取最具代表性的几个 信息: 尺寸、公差和表面粗粘度

$$
P=\left\{d_{i}, t_{i}, r_{i}\right\}
$$

式中, $P$ 为依附于第 $i$ 道和 $i+1$ 道工序模型间的几何 演变 $G$ 的工艺语义信息; $d_{i}$ 为几何演变的最大尺寸, 反映加工特征的几何尺度信息; $t_{i}$ 为加工特征各面 公差等级的最高值; $r_{i}$ 为加工特征的表面粗粘度。

$G$ 的捕捉、降维处理和后文加工意图几何演变的 捕捉、降维处理方法相同。 $P$ 的获取、降维处理和后 文加工意图工艺语义的获取、降维处理方法相同。

对于 MBD 工艺知识元模型中的信息，可以分 别通过工艺知识网和关系数据库的模式进行存储和 管理。文献[6]中借鉴复杂网络的相关理论和方法对 工序模型的几何演变信息进行了存储和管理, 其所 建立的工艺知识网在本质上就等同于工艺知识库。 本文分别应用文献 [6]中方法和关系数据库来对 PKU 中的 $G$ 和 $P$ 进行存储, 这里不再赘述。

\section{2 加工意图}

加工意图(Machining intent, MI)是工艺员在拿到 一个新零件后对其工艺设计过程产生的大体设计思 路, 是对零件关键尺寸加工顺序安排的思考。加工意 图反映了零件关键尺寸的加工过程, 可以被看作是后 续进行零件详细工艺设计时的一个参照 “草稿”。

即使面对相同的零件，不同的工艺员可能会有 不同的加工意图，不同的加工意图蕴含着不同的工 艺设计思路，而不同的工艺设计思路在工艺知识重 用时就会有不同的知识需求。因此, 基于加工意图 的工艺知识重用过程强化了工艺员的主观能动性, 反映了工艺员对于工艺知识的个性化、多元化需求, 是更贴近工艺知识检索需求的新模式。

\section{3 基于加工意图的工艺知识重用方法}

加工意图是工艺员抽象的工艺设计思维, 具有 
高维、隐性、抽象等特点, 要实现基于加工意图的 工艺知识重用, 首先就需要对加工意图进行建模, 构建出高维的加工意图模型; 其次, 对高维的加工 意图模型和 MBD 工艺知识元进行降维处理, 获得 低维、可比较的形式; 最后, 建立低维的加工意图 模型与 MBD 工艺知识元间的相似性度量算法, 实 现工艺知识的检索重用。

\section{2 加工意图的建模及降维处理}

\subsection{MBD 工艺知识元加工意图的建模}

加工意图建模方法: 在空间维度上构建加工特 征语义标注模式; 在时间维度上构建加工特征序列 语义标注模式。

（1）在空间维度上构建加工特征语义标注模 式。在空间维度上构建加工特征语义标注是为了反 映工艺人员对保证零件关键尺寸加工状态的构思。

定义 1: 关键工序模型(Key step process model, $\mathrm{KPM})$, 是指零件从开始加工到加工完成过程中所经 过的中间状态的三维模型, 这里的加工过程是工艺 员对零件加工过程的大体构思。

在关键工序模型中, 尺寸信息反映零件关键尺 寸的加工状态, 而公差/表面粗粘度则与制造特征加 工方法的选择密切相关。因此, 选择零件关键尺寸 及其公差和表面粗粘度加工要求作为关键工序模型 的工艺语义信息。关键工序模型由工艺员根据自己 的加工意图确定, 以人机交互的方式输入到三维 $\mathrm{CAD}$ 系统中。

（2）在时间维度上构建加工特征序列语义标注 模式。

在时间维度上构建加工特征序列语义标注模 式, 即构建关键工序模型序列, 是为了反映工艺人 员对零件关键尺寸加工先后次序安排的构思。不同 的加工意图体现为不同的关键工序模型序列, 而不 同的关键工序模型序列映射为不同的加工意图。将 关键工序模型序列(Model sequence, MS)表示为

$$
\mathrm{MS}=\left\{M_{1}, M_{2}, \cdots, M_{k}\right\}
$$

式中, $k$ 为关键工序模型序列中包含的模型个数; $M_{1} 、 M_{2} 、 \cdots 、 M_{k}$ 为关键工序模型序列中的关键 工序模型。

工艺人员根据自身的加工意图, 以人机交互的 方式在三维 CAD 系统中完成对 $\mathrm{MS}$ 的构建。

\section{2 高维加工意图检索模型}

工艺员的加工意图蕴含在关键工序模型序列的 动态演变过程中, 模型序列的几何演变信息、几何
演变的工艺语义信息一起构成具体的、形象化后的 高维加工意图。高维加工意图(High-dimensional machining intent, HMI)的矢量表达形式

$$
\mathrm{HMI}=\left[G_{H}, P_{H}\right]
$$

或

$$
\mathrm{HMI}=\left[G_{H i}, P_{H i} \mid i=1,2, \cdots, k-1\right]
$$

式中, $G_{H}$ 为关键工序模型序列的几何演变集合; $P_{H}$ 为关键工序模型序列的几何演变对应的工艺语义信 息集合。

$$
G_{H}=\left\{G_{H 1}, G_{H 2}, \cdots, G_{H k-1}\right\}
$$

式中, $G_{H i}(1 \leqslant i \leqslant k-1)$ 为模型序列中相邻两个关键 工序模型间的几何演变信息。

$$
P_{H}=\left\{P_{H 1}, P_{H 2}, \cdots, P_{H k-1}\right\}
$$

式中, $P_{H i}=\left\{D_{i}, T_{i}, R_{i}\right\}(1 \leqslant i \leqslant k-1)$ 是依附于 $G_{H i}$ 的工 艺语义信息; $P_{H i}$ 中的包含三个元素: $D_{i}$ 为零件的 关键尺寸, $T_{i}$ 是对应尺寸的公差要求, $R_{i}$ 为该道工 序的加工粗粘度要求。

\section{3 高维加工意图的降维处理}

对 HMI 进行降维处理, 使之转变为可以与 MBD 工艺知识元进行比较的形式, 是进行工艺相似 性检索和重用的前提。而 HMI 降维处理的前提是准 确捕捉到工艺员加工意图模型中的几何演变信息和 工艺语义信息。

\subsection{1 高维加工意图的几何演变、工艺语义捕捉}

(1) 几何演变捕捉。

几何演变的捕捉方法: 将关键工序模型中相邻 两个模型存为 STL 格式; 然后以重建三角面片拓扑 关系的 STL 文件为数据源, 应用改进形状分布算法 $D_{1}$ 距离来构建模型各表面的形状分布直方图; 接着 对直方图进行保形插值, 拟合得到形状分布曲线; 最后通过对曲线进行相似性度量来获取相邻两个模 型的几何演变。对 MS 中的所以前、后相邻工序模 型重复上述方法, 即可得到高维加工意图的几何演 变信息集合。

STL 文件简单, 易读取, 且其表示模型不失真, 有较高精度。当前流行的 CAD 软件几乎都具备 STL 文件接口 ${ }^{[9]}$, 通过在 $\mathrm{CAD}$ 系统中进行相应的设置, 就可以获得与模型逼近精度较高的 STL 文件。因此, 本文在用形状分布算法表征模型表面形状分布时, 以 STL 文件作为数据来源。但是由于 STL 文件数据 的午余性和面片的无序性 ${ }^{[10]}$, 本文在对 STL 文件中 的三角面片拓扑关系进行重建的基础上，提出了改 进形状分布算法 $D_{1}$ 距离来构建关键工序模型各表 
面的形状分布直方图。

STL 文件中三角面片拓扑关系的建立方法：当 模型表面是平面时, 寻找法矢相同且共边的三角面

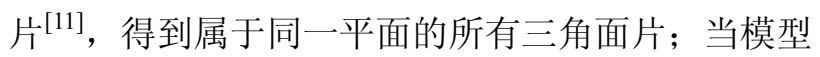
表面不是平面时, 计算每个三角面片和与其共边的 3 个三角面片的法矢夹角, 若角度小于某一阈值(一 般选阈值为 $20^{\circ}$ ), 则认为该相邻面片与此面片共 面, 否则不共面 ${ }^{[8]}$; 按照上述方法遍历模型所有表 面, 得到模型每个面包含的所有三角面片。

改进形状函数 $D_{1}$ 距离: STL 文件中三角面片内 心到模型形心的距离。改进形状函数 $D_{1}$ 距离避免了 因为数据冗余而造成的 STL 文件处理过程较复杂且 耗时的缺陷。

在模型表面的形状直方图中, 横坐标取 STL 模 型表面的所有三角面片的改进形状函数 $D_{1}$ 距离或 距离区间, 纵坐标取某一距离或距离区间内包含的 三角面片内心的频数。对直方图保形插值拟合得到 形状分布曲线, 通过曲线的相似性度量, 计算得到 两个三维模型对应表面的相似性, 从而得到相邻两 个关键工序模型间的几何演变。曲线的相似性度量 不作为本文的研究内容, 这里不再赘述。

(2) 工艺语义捕捉。

HMI 的工艺语义信息捕捉通过 CAD 系统的应 用程序接口来实现。

2.3.2 高维加工意图几何演变、工艺语义的降维处理 几何演变、工艺语义的降维处理过程如图 2 所示。

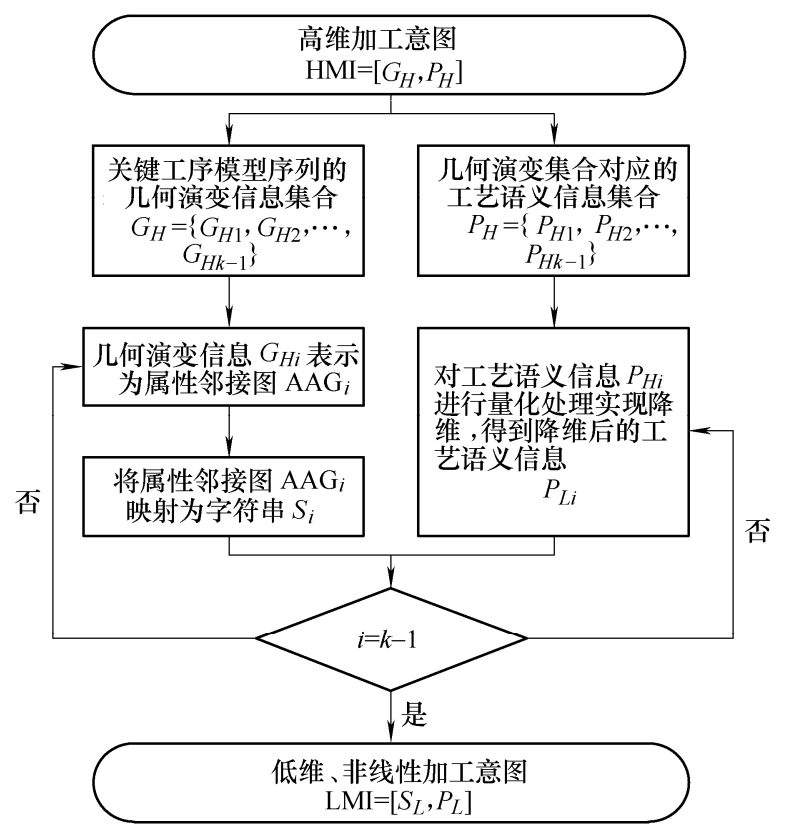

图 2 高维加工意图的降维处理

(1) 几何演变的降维处理。

用属性邻接图 $\mathrm{AAG}=\{V, E, \alpha, \beta, \gamma\}$ 对零件模型
的几何拓扑信息进行描述。其中, $V$ 为图中节点的 集合, 节点代表模型的表面, 对于模型的任意一个 表面 $f_{i}$, 都有唯一的节点 $n_{i} \in V$ 与之对应; $E$ 为图 中弧(或边)的集合, 弧(或边)代表表面之间的邻接 关系, $f_{i} 、 f_{j}$ 相邻, 弧为实线, $f_{i} 、 f_{j}$ 不相邻, 弧为 虚线, 对于模型中任意的一对表面 $f_{i} 、 f_{j}$, 都有唯 一的弧 $e_{i} \in E$ 与之对应; $\alpha$ 代表模型中面的属性集, 如面的类型(平面、圆柱面等)、面积、法矢等; $\beta$ 表示模型中边的属性集，包括边的类型(直线、圆 弧等)、长度等; $\gamma$ 表示几何对象的关联属性集, 如 两个面相邻、平行。图 3 是一个轴零件的属性邻接 图描述实例。

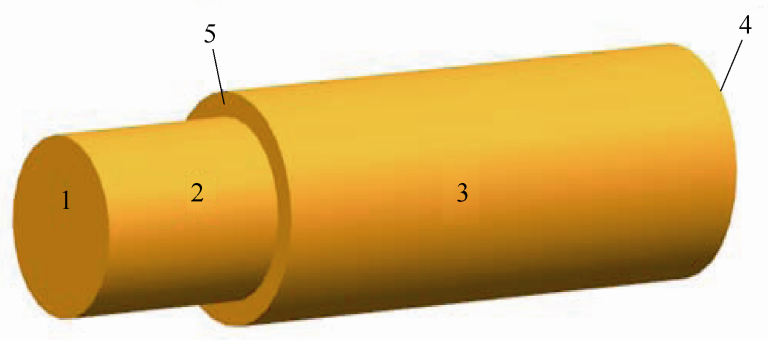

(a) 轴的三维模型

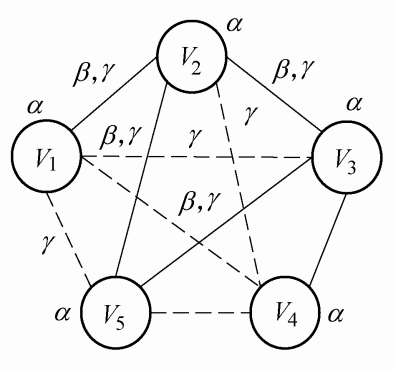

(b) 轴三维模型的属性邻接图

图 3 轴零件的属性邻接图表示

在获得零件模型的属性邻接图后, 就可以应用 图的相似性度量算法来比较零件几何结构信息的相 似性。传统属性邻接图的相似性比较方法主要有基 于图的邻接矩阵和基于图的编辑距离等方法。基于 图的邻接矩阵的算法比较效果不好 ${ }^{[12]}$, 而计算图的 编辑距离又是一个 NP-Hard 问题 ${ }^{[13]}$ 。近年来, 基于 字符串相似性度量的比较方法成为了研究热点, 取 得很多研究成果, 如基于编辑距离的相似性度量方 法 $^{[14]}$ 。考虑到度量字符串的相似性比直接度量图的 相似性要简单, 所以本文先将属性邻接图映射转换 为具有唯一性的字符串, 然后通过度量字符串的相 似性来完成对几何演变相似性的计算。

属性邻接图映射为字符串的方法: 根据三维模 型的属性定义一个排序函数, 对属性邻接图中的顶 点排序, 得到顶点的唯一排序序列; 对顶点间的关 
系集进行定义, 并进一步将顶点排序后的属性邻接 图表示为字符串 ${ }^{[15]}$ 。

(2) 工艺语义的降维处理。

工艺语义信息的降维处理通过对其量化处理来 实现。以尺寸公差为例, 其量化处理可根据获取到 的尺寸公差来确定其公差等级：然后对照标准公差 数值表, 根据公称尺寸和公差值来反推得到标准公 差等级。

\subsection{3 低维非线性加工意图}

按照前两节给出的方法, 对 HMI 的几何演变和 工艺语义信息分别进行降维处理, 得到低维、非线 性加工意图。参照 MBD 工艺知识元和 HMI 的矢量 表达给出低维、非线性加工意图(Lower-dimensional machining intent, LMI)的矢量表达

$$
\mathrm{LMI}=\left[S_{L}, P_{L}\right]
$$

或

$$
\mathrm{LPI}=\left[\left(S_{L 1}, P_{L 1}\right),\left(S_{L 2}, P_{L 2}\right), \cdots,\left(S_{L k-1}, P_{L k-1}\right)\right]
$$

式中, $S_{L}$ 为表达关键工序模型序列几何演变信息的 字符串集合; $S_{L i}$ 为字符串集合中的第 $i$ 个字符串, 表达第 $i$ 个和第 $i+1$ 个关键工序模型间的几何演变; $P_{L}$ 为降维后的工艺语义信息集合; $P_{L i}$ 为 $P_{L}$ 中的第 $i$ 个元素，表达 $S_{L i}$ 对应的工艺语义信息。

$$
\begin{gathered}
S_{L}=\left\{S_{L 1}, S_{L 2}, \cdots, S_{L k-1}\right\} \\
P_{L}=\left\{P_{L 1}, P_{L 2}, \cdots, P_{L k-1}\right\} \\
P_{L i}=\left\{D_{L i}, T_{L i}, R_{L i}\right\} \quad 1 \leqslant i \leqslant k-1
\end{gathered}
$$

在 $P_{L i}$ 中, $T_{L i}$ 为高维加工意图中的尺寸公差值; $T_{H i}$ 对应的尺寸公差等级。

\section{3 加工意图与 MBD 工艺知识元的相 似性度量}

基于加工意图的工艺知识重用就是将加工意图 作为检索条件, 以工艺知识库中的 MBD 工艺知识 元作为检索对象, 通过二者之间的相似性度量来实 现检索重用。低维、非线性的加工意图 LMI 与 MBD 知识元间的相似性度量可以简单描述为: 遍历 $\mathrm{LMI}=\left[S_{L}, P_{L}\right]$ 或 $\mathrm{LMI}=\left[S_{L i}, P_{L i} \mid i=1,2, \cdots, k-1\right]$ 的 每一项 $I_{i}$ (记 $I_{i}=\left\{S_{L i}, P_{L i}\right\}$ ), 并用其在工艺知识库中 进行检索, 得到与之最相似 MBD 工艺知识元。本 文只给出 LMI 中的单项 $I_{i}=\left\{S_{L i}, P_{L i}\right\}$ 与单个 MBD 工艺知识元的相似性度量方法, 重复应用相同的方 法就可以得到与 LMI 中所有项都匹配的 MBD 工艺 知识元集合, 即工艺知识库中和 LMI 相似的所有
MBD 工艺知识元。

LMI 单项 $I_{i}$ 与 MBD 工艺知识元的相似性度量 分为几何演变信息的相似性度量和工艺语义信息的 相似性度量两部分。

\section{1 几何演变信息的相似性度量}

通过降维处理 MBD 工艺知识元的几何演变 信息, 使其转化为可以和 LMI 进行相似性比较的 字符串。编辑距离算法通常被用于字符串的快速 匹配, 是一种经典而广泛使用的方法 ${ }^{[16]}$ 。但是, 由于本文要进行相似性度量的字符串包含了几 何演变的几何和拓扑信息, 原始的编辑距离并不 适用。因此, 本文采用改进编辑距离字符串相似 性度量方法 ${ }^{[15]}$ 来实现对几何演变信息的相似性 度量。

为了便于描述基于改进编辑距离的字符串相似 性度量方法, 将 $I_{i}$ 中表示几何演变的字符串 $S_{L i}$ 记为 $S_{1}$, 将表示 MBD 工艺知识元中几何演变的字符串 $S$ 记为 $S_{2}$, 字符串 $S_{1} 、 S_{2}$ 的长度分别为 $m 、 n$ 。建立 $S_{1} 、 S_{2}$ 的关系矩阵 $E D=\left[D_{i j}\right]_{(m+1) \times(n+1)}$ 。 $D_{i j}$ 可以根据 下式进行迭代计算

$$
D_{i j}=\min \left\{\begin{array}{l}
D(i-1, j-1)+R\left(S_{1}\langle i\rangle \rightarrow S_{2}\langle j\rangle\right) \\
D(i-1, j)+R\left(S_{1}\langle i\rangle \rightarrow \Lambda\right) \\
D(i, j-1)+R\left(\Lambda \rightarrow S_{2}\langle j\rangle\right)
\end{array}\right.
$$

式中, $S_{1}\langle i\rangle$ 为字符串 $S_{1}$ 中的第 $i$ 个字符; $S_{2}\langle j\rangle$ 为字符串 $S_{2}$ 中的第 $j$ 个字符; $R$ 表示代价函数, $R\left(S_{1}\langle i\rangle \rightarrow S_{2}\langle j\rangle\right)$ 代表将字符串 $S_{1}$ 中的第 $i$ 个字符 $S_{1}\langle i\rangle$ 用符串 $S_{2}$ 中的第 $j$ 个字符 $S_{2}\langle j\rangle$ 替换付出的代 价, $R\left(S_{1}\langle i\rangle \rightarrow \Lambda\right)$ 代表删除字符串 $S_{1}$ 中的第 $i$ 个字 符 $S_{1}\langle i\rangle$ 所付出的代价, $R\left(\Lambda \rightarrow S_{2}\langle j\rangle\right)$ 表示字符串 $S_{1}$ 中插入字符串 $S_{2}$ 中的第 $j$ 个字符 $S_{2}\langle j\rangle$ 所付出的 代价。

在迭代得到字符串 $S_{1}$ 和 $S_{2}$ 间编辑距离 $D_{m n}$ 后, 就可以利用式(13) ${ }^{[17]}$ 来度量字符串间的相似度。

$$
\operatorname{Sim}\left(S_{1}, S_{2}\right)=1-\frac{D_{m n}}{m+n}
$$

$0 \leqslant \operatorname{Sim}\left(S_{1}, S_{2}\right) \leqslant 1, \quad \operatorname{Sim}\left(S_{1}, S_{2}\right)=0$ 表示 $S_{1}$ 和 $S_{2}$ 完全不相似, $\operatorname{Sim}\left(S_{1}, S_{2}\right)=1$ 表示 $S_{1}$ 和 $S_{2}$ 完全相 等。字符串 $S_{1}$ 和 $S_{2}$ 的相似度也就反映了 LMI 和 MBD 工艺知识元中的几何演变信息的相似度。

\section{2 工艺语义信息的相似性度量}

$\mathrm{LMI}$ 单项 $I_{i}=\left\{S_{L i}, P_{L i}\right\}$ 中的工艺语义信息 $P_{L i}$ 和 MBD 工艺知识元中工艺语义信息 $P$ 的相似性包括 尺寸、尺寸公差以及表面粗楉度的相似性这三个部 
分。三者的相似度 $\operatorname{Sim}\left(D_{L i}, d\right) 、 \operatorname{Sim}\left(T_{L i}, t\right)$ 、 $\operatorname{Sim}\left(R_{L i}, r\right)$ 都可根据下式计算

$$
\operatorname{Sim}\left(x_{1}, x_{2}\right)=1-\frac{\left|x_{1}-x_{2}\right|}{\max \left(x_{1}, x_{2}\right)}
$$

式中, $x_{1}$ 表示 $P_{L i}$ 中的尺寸值、尺寸公差等级、表 面粗粘度值; $x_{2}$ 表示工艺知识元中 $P$ 包含的尺寸值、 尺寸公差等级、表面粗楉度值。

和 $\operatorname{Sim}\left(S_{1}, S_{2}\right)$ 的性质类似, 尺寸、尺寸公差以 及表面粗糙度的相似性也满足: $0 \leqslant \operatorname{Sim}\left(D_{L i}, d\right)$, $\operatorname{Sim}\left(T_{L i}, t\right), \operatorname{Sim}\left(R_{L i}, r\right) \leqslant 1$ 。

\section{3 多属性融合的工艺知识元相似性度量}

综合考虑几何演变信息的相似性和工艺语义信 息的相似性, 给出多属性融合的 LMI 与 MBD 工艺 知识元间的相似性加权评价模型。LMI 单项 $I_{i}$ 与 MBD 工艺知识元 $P K U$ 的相似度记为 Sim。

$$
\begin{aligned}
\operatorname{Sim}= & w_{G} \operatorname{Sim}\left(S_{L i}, S\right)+w_{D} \operatorname{Sim}\left(D_{L i}, d\right)+ \\
& w_{T} \operatorname{Sim}\left(T_{L i}, t\right)+w_{R} \operatorname{Sim}\left(R_{L i}, r\right)
\end{aligned}
$$

式中, $\operatorname{Sim}\left(S_{L i}, S\right)$ 表示 $I_{i}$ 中的字符串 $S_{L, i}$ 与 PKU 中
的字符串 $S$ 的相似度; $\operatorname{Sim}\left(D_{L i}, d\right), \operatorname{Sim}\left(T_{L i}, t\right)$, $\operatorname{Sim}\left(R_{L i}, r\right)$ 分别为 $I_{i}$ 和 PKU 中包含的尺寸、尺寸公 差以及表面粗䊁度等信息的相似度; $w_{G}, w_{D}, w_{T}$, $w_{R}$ 分别为 $\operatorname{Sim}\left(S_{L i}, S\right), \operatorname{Sim}\left(D_{L i}, d\right), \operatorname{Sim}\left(T_{L i}, t\right)$, $\operatorname{Sim}\left(R_{L i}, r\right)$ 对应的权重。关于权重设置的一个启发 式原则是高层制造语义信息不小于低层的几何形状 信息 ${ }^{[18]}$, 即 $w_{T}+w_{R} \geqslant w_{G}+w_{D}$, 且 $\sum_{T R G D} w=1$ 。各 个权重的具体取值需要根据不同企业工艺设计工作 的经验来确定。

同样的, $0 \leqslant \operatorname{Sim} \leqslant 1$ 。给定一个阈值 $\varepsilon$, 若 $\operatorname{Sim}>\varepsilon$, 则认为 $I_{i}$ 与 PKU 相似; 否则, 不相似。

应用式(15)对 LMI 中的每一项与工艺知识库 中的 MBD 工艺知识元进行相似性度量, 就可得到 与 LMI 相似的 MBD 工艺知识元的集合, 即 $\left\{\mathrm{PKU}_{1 q_{1}}, \mathrm{PKU}_{2 q_{2}}, \cdots, \mathrm{PKU}_{k-1 q_{k-1}}\right\}$ 。

对于 $\mathrm{PKU}_{i j}\left(i=1,2, \cdots, k-1 ; j=1,2, \cdots, q_{i}\right), q_{i}$ 为 和 LMI 分量 $I_{i}$ 相似的 MBD 工艺知识元的个数。 LMI 与 MBD 工艺知识元的相似性度量过程如图 4 所示。

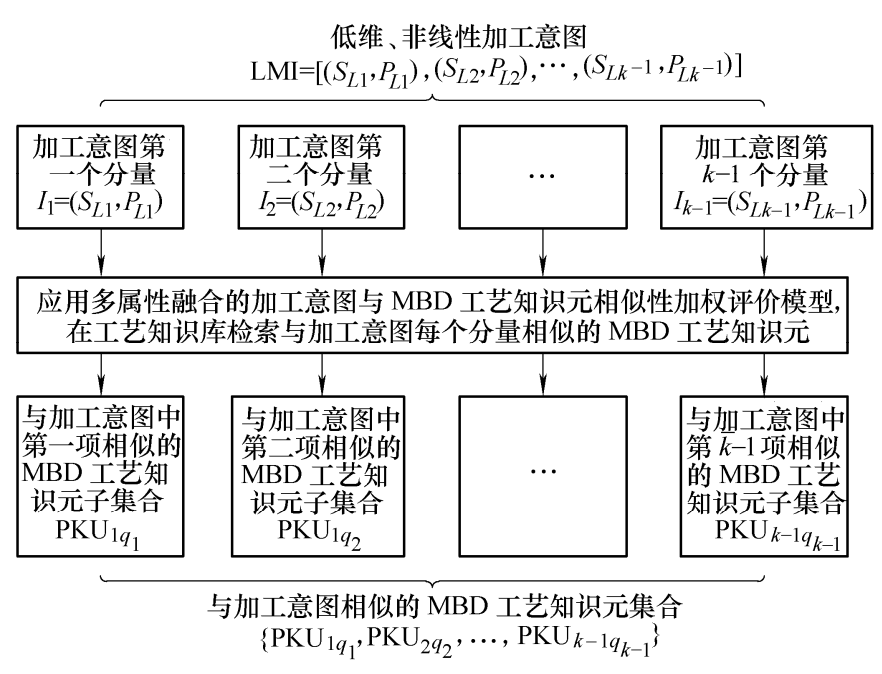

图 4 LMI 与 MBD 工艺知识元的相似性度量过程

\section{4 实例验证}

阶梯轴零件是机械产品中的常用零件, 其加工 过程更是包含了车削、铣削、钻削和磨削等常见的 机加工序, 其工艺过程具有典型性和代表性。因此, 本文选择阶梯轴零件作为研究对象来进行实例验 证, 如图 5 所示。图中未注明公差都采用标准公差 IT7。

设某工艺员根据自己对图 5 所示阶梯轴零件 的加工意图, 构建出了如图 6 所示的关键工序模型 序列。

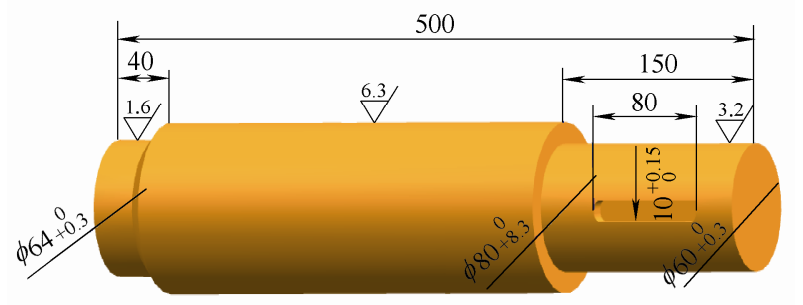

图 5 阶梯轴

使用第 2.3.1 节的方法捕捉图 6 中关键工序模型 序列的几何演变; 使用第 2.3.2 节的方法将几何演变 
集合中的元素分别表示为属性邻接图, 并应用排 序函数 $R(V)$ 对各属性邻接图的顶点重新排序, 结 果如图 7 所示。其中, $G_{H 1} 、 G_{H 2} 、 G_{H 3}$ 分别表示 图 6a、6b间, 图 6b、6c 间, 图 6c、6d 间的几何

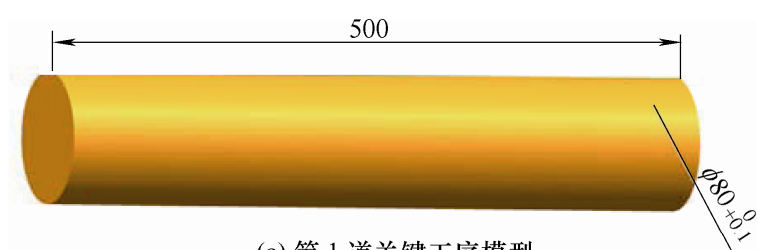

(a) 第 1 道关键工序模型

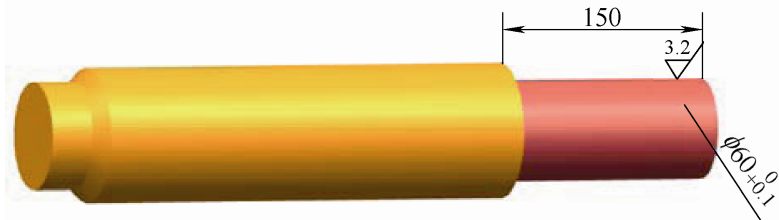

(c) 第 3 道关键工序模型
演变; $G_{a 1} 、 G_{b 1} 、 G_{c 1}$ 分别是对几何演变 $G_{H 1} 、 G_{H 2}$ 、 $G_{H 3}$ 的属性邻接图表示; $G_{a 2} 、 G_{b 2} 、 G_{c 2}$ 分别是对 $G_{a 1} 、 G_{b 1} 、 G_{c 1}$ 的顶点进行重新排序后生成的新属 性邻接图。

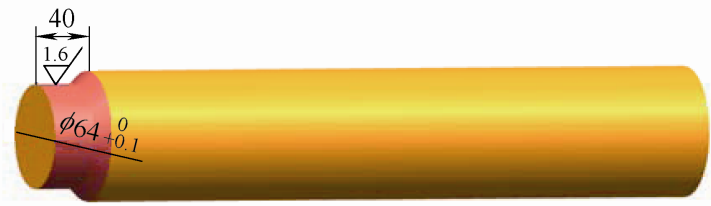

(b) 第 2 道关键工序模型

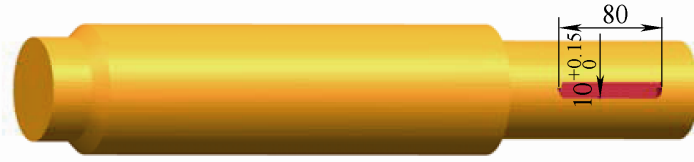

(d) 第 4 道关键工序模型

图 6 关键工序模型序列

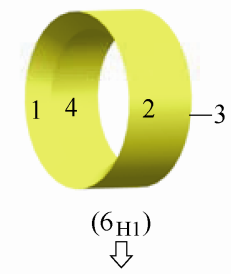

$(B, 7540)$

$b, \gamma$ (V) $b, \gamma$

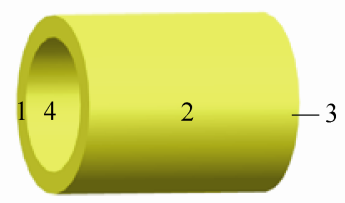

$\left(6 \mathrm{H}_{2}\right)$

\

$(B, 37699)$

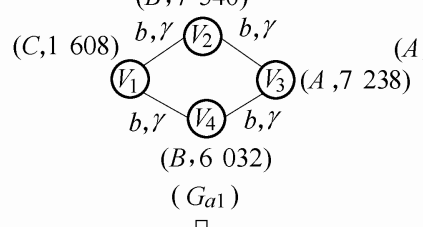

$\Omega$

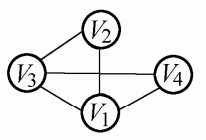

$(G a 2)$ $b, \gamma$ (1/2) $b, \gamma_{(A, 8796)}$

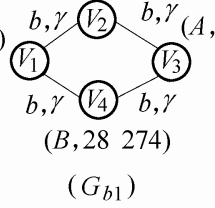

万

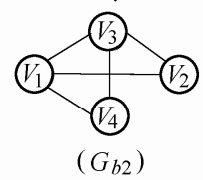

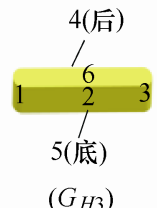

(A 560$)$
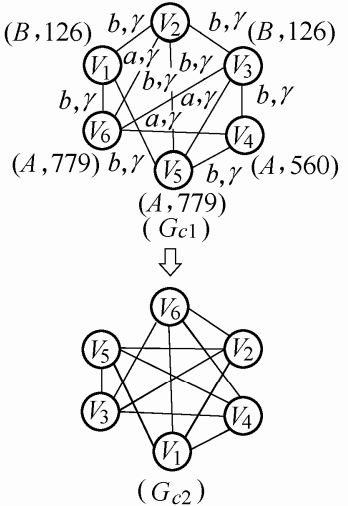

图 7 关键工序模型序列几何演变的属性邻接图表示

使用第 2.3.1 节的方法捕捉图 6 中模型序列的工 艺语义信息。使用第 2.3.2 节的方法对捕捉到的工艺 语义信息进行降维处理。结果如表 1 所示。

\section{表 1 几何演变对应的工艺语义信息}

\begin{tabular}{cccc}
\hline \multirow{2}{*}{ 几何演变 } & \multicolumn{3}{c}{ 几何演变对应的工艺语义信息 } \\
\cline { 2 - 4 } & 尺寸 $/ \mathrm{mm}$ & 精度 $(\mathrm{IT})$ & 表面粗粘度/ $\mu \mathrm{m}$ \\
\hline$G_{H 1}$ & 80 & 7 & 1.6 \\
$G_{H 2}$ & 150 & 7 & 3.2 \\
$G_{H 3}$ & 80 & 7 & 3.2 \\
\hline
\end{tabular}

最终得到的低维、非线性加工意图表示为

$$
\begin{gathered}
\mathrm{LMI}=\left[\left(S_{L 1}, P_{L 1}\right),\left(S_{L 2}, P_{L 2}\right),\left(S_{L 3}, P_{L 3}\right)\right] \\
S_{L}=\left\{S_{L 1}, S_{L 2}, S_{L 3}\right\}=\{A b B B \infty B B b C,
\end{gathered}
$$$$
A \propto A A b B B \propto B, A \infty A A a A A \infty A A a B B \infty B\}
$$

$$
P_{L}=\left\{P_{L 1}, P_{L 2}, P_{L 3}\right\}
$$

这里仅以 $\mathrm{LMI}$ 中的第一个分量 $\left(S_{L 1}, P_{L 1}\right)$ 为例来 进行相似性度量的验证。设要和 LMI 的第一个分量 进行相似性度量的 MBD 工艺知识元为

$$
\begin{gathered}
\mathrm{PKU}=[I D, G, P, Q]= \\
{[I D, A a B B b B B b C,(60,6,3.2), Q]}
\end{gathered}
$$

按照式(13)计算得到 $\operatorname{Sim}\left(S_{L i}, S\right)=87.2 \%$, 按照式 (14)计算得 $\operatorname{Sim}\left(D_{L i}, d\right)=75 \%, \operatorname{Sim}\left(T_{L i}, t\right)=85.7 \%$, $\operatorname{Sim}\left(R_{L i}, r\right)=50 \%$ 。

根据式(15)计算式(16)中 LMI 的第一个分量 $\left(S_{L 1}, P_{L 1}\right)$ 和式(19)MBD 工艺知识元 PKU 的相似度。 取 4 个分量的权重分别为: $w_{G}=0.3, w_{D}=0.2$, $w_{T}=0.3, w_{R}=0.2$ 。 


$$
\begin{gathered}
\operatorname{Sim}=w_{G} \operatorname{Sim}\left(S_{L i}, S\right)+w_{D} \operatorname{Sim}\left(D_{L i}, d\right)+ \\
w_{T} \operatorname{Sim}\left(T_{L i}, t\right)+w_{R} \operatorname{Sim}\left(R_{L i}, r\right)=76.9 \%
\end{gathered}
$$

给定阈值 $\varepsilon$ 为 $65 \%, \operatorname{Sim}>\varepsilon$ 。此判定 $\left(S_{L 1}, P_{L 1}\right)$ 和给定的 PKU 相似。

\section{5 结论}

(1) 以 MBD 工序模型几何演变过程中蕴含的 工艺知识为研究对象, 考虑了动态、关联的演变过 程中的知识, 从加工过程的几何演变及其所附带的 工艺语义这两个角度给出了 MBD 工艺知识元的表 达形式，解决了工艺知识粒度大的问题。

(2) 对加工意图进行了建模, 并提出了基于加工 意图的工艺知识重用方法, 从而有效强化了工艺员 的主观能动性在工艺知识重用过程中的作用, 更好 地满足了工艺员对于知识的个性化和多元化需求。

(3) 提出了加工意图与 MBD 工艺知识元间相 似性度量方法, 实现了对工艺知识的检索和重用。

\section{参 考 文 献}

[1] 刘书暖. 工艺知识发现与重用技术[D]. 西安: 西北工业 大学, 2007

LIU Shunuan. Process knowledge discovery and reuse technology[D]. Xi'an : Northwestern Polytechnical University, 2007.

[2] 皇甫中民, 张树生, 间雒恒. 鱼群启发的三维 CAD 模 型聚类与检索 $[\mathrm{J}]$. 计算机辅助设计与图形学学报, 2016, 28(8): 1373-1382

HUANGFU Zhongmin, ZHANG Shusheng, YAN Luoheng. 3D CAD model clustering and retrieval inspired by fish swarm[J]. Journal of Computer-Aided Design \& Computer Graphics， 2016， 28(8): 1373-1382.

[3] 伅铮. 基于图论的三维 CAD 模型检索技术研究 [D]. 哈 尔滨: 哈尔滨理工大学, 2014.

ZHANG Zheng. 3D CAD model retrieval based on graph theory[D]. Harbin: Harbin University of Science and Technology, 2014.

[4] CHANG H C, DONG L, LIU F X, et al. Indexing and retrieval in machining process planning using case-based reasoning[J]. Artificial Intelligence in Engineering, 2000, 14(1): $1-13$.

[5] 胡淑慧, 杨海成, 张招建, 等. 三维工序模型几何演变 序列的相似性度量 $[\mathrm{J}]$. 计算机辅助设计与图形学学报, 2014, 26(7): 1176-1183.

HU Shuhui, YANG Haicheng, ZHANG Zhaojian, et al. Simi-larity measure of the geometry change sequence of three-dimensional process model[J]. Journal of
Computer-Aided Design \& Computer Graphics, 2014, 26(7): 1176-1183.

[6] 李春磊, 莫蓉, 常智勇, 等. 几何演变驱动的机加工艺 知识表示与推送 $[\mathrm{J}]$. 计算机集成制造系统, 2016, 22(6): $1434-1446$

LI Chunlei, MO Rong, CHANG Zhiyong, et al. Knowledge representation and push of machining process driven by geometric variation[J]. Computer Integrated Manufacturing Systems, 2016, 22(6): 1434-1446.

[7] 王英伟, 莫蓉, 万能. 多粒度机械加工设计意图建模方 法研究 [J]. 工具技术，2014，48(6): 31-35.

WANG Yingwei, MO Rong, WAN Neng. Multigranularity modeling method of machining design intent[J]. Tool Engineering, 2014, 48(6): 31-35.

[8] 刘晨, 殷国富, 龙红能. 制造工艺知识粒度描述方法与 获取算法研究 [J]. 计算机集成制造系统, 2008，14(10): 1966-1973.

LIU Chen, YIN Guofu, LONG Hongneng. Description and discovery method of manufacture process knowledge granularity $[\mathrm{J}]$. Computer Integrated Manufacturing Systems, 2008, 14(10): 1966-1973.

[9] 肖棋，林俊义. STL 文件格式在反求造型中的应用 $[\mathrm{J}]$. 华侨大学学报，2006，27(3): 284-287.

XIAO Qi, LIN Junyi. The application of STL file format in reverse engineering $[\mathrm{J}]$. Journal of Huaqiao University, 2006, 27(3): 284-287.

[10] 纪峰, 李占利. 基于 STL 文件的拓扑重构新方法 [J]. 湖 南科技学院学报，2006，27(5): 154-157.

JI Feng, LI Zhanli. A novel method for the topological reconstruction based on STL files[J]. Journal of Hunan University of Science \& Engineering, 2006, 27(5): 154-157.

[11] 王建新, 王从军, 黄树槐. 基于 STL 文件格式的线框 模型算法的研究与实现 [J]. 机械与电子, 2002(3): 28-30.

WANG Jianxin, WANG Congjun, HUANG Shuhuai. Research and implement on the algorithms of the wireframe based on the STL format[J]. Machinery \& Electronics, 2002(3): 28-30.

[12] MCWHERTER D T, PEABODY M, REGLI W C, et al. Solid model databases: Techniques and empirical results $[\mathrm{J}]$. Journal of Computing and Information Science in Engineering, 2001, 1(4): 300-310.

[13] BUNKE H. On a relation between graph edit distance and maximum common subgraph[J]. Pattern Recognition Letters, 1997, 18(9): 689-694.

[14] WAGNER R A, FISCHER M J. The string-to-string correction problem[J]. Journal of the ACM，1974，21(1): 168-173. 
[15] 张欣, 莫蓉, 石源, 等. 基于字符串度量的 CAD 模型 相似性比较算法 $[\mathrm{J}]$. 中国机械工程, 2009, 20(20): 2435-2440.

ZHANG Xin, MO Rong, SHI Yuan, et al. String-based CAD models similarity assessment algorithm[J]. China Mechanical Engineering, 2009, 20(20): 2435-2440.

[16] 姜华, 韩安琪, 王美佳, 等. 基于改进编辑距离的字符串 相似度求解算法 [J]. 计算机工程, 2014，40(1): 222-227 JIANG Hua, HAN Anqi, WANG Meijia, et al. Solution algorithm of string similarity based on improved levenshtein distance[J]. Computer Engineering, 2014, 40(1): 222-227.

[17] 周汉平. Levenshtein 距离在编程题自动评阅中的应用 研究[J]. 计算机应用与软件, 2001，28(5)：209-212.
ZHOU Hanping. Study on application of levenshtein distance in programming test automatic scoring $[\mathrm{J}]$. Computer Applications \& Software, 2011, 28(5): 209-212.

[18] 黄瑞, 张树生, 白晓亮, 等. 融合制造语义的三维 CAD 模型检索方法[J]. 计算机集成制造系统，2013，19(6): 1177-1185.

HUANG Rui, ZHANG Shusheng, BAI Xiaoliang, et al. Manufacturing semantics based 3D CAD model retrieval method[J]. Computer Integrated Manufacturing Systems, 2013, 19(6): 1177-1185.

作者简介: 常智勇(通信作者), 男, 1971 年出生, 博士，副教授，硕士 研究生导师。主要研究方向为网络 CAD、CAGD、数字化制造。

E-mail: changzy@nwpu.edu.cn

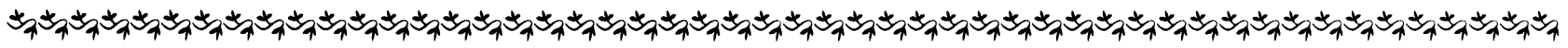

\section{第 7 届上银优秀机械博士论文奖——佳作奖}

作者: 张辉

\section{混合润滑状态下表面织构的建模和设计研究}

毕业学校: 西安交通大学

指导教师: 董光能 Meng HUA K S CHIN

织构化技术指的是在微纳米尺度下, 通过物理或化学方法在机械滑动支承表面加工凹坑、沟槽或凸起结构。近二十年来 的研究表明, 织构化表面可以显著改善机械配副的摩擦学特性, 因此工程中得到了广泛应用。然而, 现行的织构设计方法往 往基于试错法或经验。对于织构设计准则, 特别是在混合润滑状态下织构设计准则相当缺失。相关文献中几乎找不到公认的 织构设计准则。因此, 本文旨在于建立计算凹形织构在混合润滑状态下摩擦学特性的数学模型, 进而研究织构非规则形状和 排布对其摩擦学特性的影响。

通过用平均流量雷诺方程和 K-E 弹塑性接触模型分别计算凹形织构化表面的流体动压作用和微凸体接触状态, 由此建立 织构混合润滑模型。并通过实验验证了该模型的有效性。利用该混合润滑模型计算圆坑织构的摩擦系数, 得到了相应的斯特 利贝克 (Stribeck) 曲线。计算结果证明了凹形织构化表面具有减小摩擦和磨损的作用。织构产生的流体动压效应, 有助于增 加油膜厚度, 从而减少微凸体的接触, 这是织构化表面减摩作用的主要机理。研究通过对比圆形、正方形、三角形织构在几 种不同滑动方向上的摩擦学特性, 发现三角形织构在某个滑动方向下具有最小的摩擦系数。研究还探讨了圆坑织构的几何参 数如直径、深度、间距等的影响, 从而得到了在某特定工况下一系列较优的织构参数。此外, 本文还对滑移边界条件下雷诺 方程进行了改进, 强调了滑移长度随剪切率线性变化的函数关系。通过该边界滑移模型, 计算了滑移表面对径向滑动轴承摩 擦学性能的影响。

以获得优良的表面摩擦学性能为目标, 利用遗传算法分别在单方向运动和往复运动条件下对凹形织构的不规则形状进行 了优化。得到了几种典型的织构形状, 如: 子弹形和鱼形织构适于单方向运动, 椭圆形和梭形织构适于往复运动。这些优化 得到的织构形状具有优于普通圆形织构的摩擦学特性。通过对油膜流体动压云图的分析, 证明了优化得到的织构形状可以产 生更高的流体动压力, 这是它们具有更好摩擦学特性的主要机理。通过实验进一步验证了相对于普通圆形织构, 优化得到的 织构形状具有更低的摩擦系数, 并且该优势在高速低载条件下更为显著。

通过旋转运动和往复运动销盘实验得到具有五种布拉维点阵排布织构化表面的摩擦系数, 从而研究织构排布对摩擦学特 性的影响。旋转运动条件下, 六方织构排布具有最小摩擦系数。往复运动条件下, 斜方织构排布在低载高频工况下具有最低 摩擦系数。此外实验表明, 旋转对称阶数和垂直于运动方向上的织构比面积可用于描述织构排布在旋转运动和往复运动中的 作用。通过对这两个参数和不同织构排布的摩擦系数进一步对比分析, 在某种程度上解释了实验结果中可能存在的机理。

本文在织构建模和设计研究中有一定的突破, 提出了织构几何尺寸、形状、排布等相应的设计理论和方法。这为织构在 机械摩擦配副上的设计和应用, 提供了更为全面的理论支持。 